ANNALES

POLONICI MATHEMATICI

$87(2005)$

\title{
The Lojasiewicz numbers and plane curve singularities
}

\author{
by Evelia García Barroso (La Laguna), Tadeusz Krasiński (Łódź) \\ and Arkadiusz PŁoski (Kielce)
}

\begin{abstract}
For every holomorphic function in two complex variables with an isolated critical point at the origin we consider the Eojasiewicz exponent $\mathcal{L}_{0}(f)$ defined to be the smallest $\theta>0$ such that $|\operatorname{grad} f(z)| \geq c|z|^{\theta}$ near $0 \in \mathbb{C}^{2}$ for some $c>0$. We investigate the set of all numbers $\mathcal{L}_{0}(f)$ where $f$ runs over all holomorphic functions with an isolated critical point at $0 \in \mathbb{C}^{2}$.
\end{abstract}

1. Introduction. Let $f$ be a holomorphic function defined near $0 \in \mathbb{C}^{2}$, such that $f(0)=0$ and with an isolated critical point at the origin, and let $(C, 0)$ be the germ of a singular plane curve with local equation $f=0$. Set $\operatorname{grad} f=(\partial f / \partial x, \partial f / \partial y)$. The Eojasiewicz exponent $\mathcal{L}_{0}(f)$ of $f$ at 0 is defined to be the smallest $\theta>0$ such that

$$
|\operatorname{grad} f(z)| \geq c|z|^{\theta} \text { in a neighbourhood of } 0 \in \mathbb{C}^{2}
$$

with a constant $c>0$.

Teissier proved (see [T, p. 275]) that the Eojasiewicz exponent $\mathcal{L}_{0}(f)$ depends only on the topological type of the germ $(C, 0)$; more specifically, $\mathcal{L}_{0}(f)+1$ is the maximal polar invariant of $(C, 0)$. In particular $\mathcal{L}_{0}(f)$ is a rational number. In this paper we investigate the problem which rational numbers are Łojasiewicz exponents of plane curve singularities. Such numbers will be called Eojasiewicz numbers. The first result in this direction was obtained in [P1, p. 359]. Namely, each Łojasiewicz number appears in the sequence

$$
1,2,3,3 \frac{1}{2}, 4,4 \frac{1}{3}, 4 \frac{1}{2}, 4 \frac{2}{3}, 5, \ldots
$$

2000 Mathematics Subject Classification: 32S05, 14H20.

Key words and phrases: Łojasiewicz exponent, plane curve singularity, characteristic sequence, intersection multiplicity.

This research was partially supported by the Spanish Project BFM 2001-2251 (the first author) and the KBN grant No 2 P03A 01522 (the third author). 
whose terms greater than 1 are rationals of the form

$$
N+\frac{b}{a}, \quad a, b, N \in \mathbb{Z}, 0 \leq b<a<N .
$$

For a new proof of this fact see Section 6 (Proposition 6.4). Unfortunately, not all numbers in (3) are Łojasiewicz numbers. Namely, in [Ga-P] it was proved that

$$
\begin{aligned}
& \text { the terms in (3) for which } \\
& \qquad a=N-1, \quad b>1, \quad \operatorname{GCD}(a, b)=1
\end{aligned}
$$

(e.g. $4 \frac{2}{3}, 5 \frac{3}{4}, \ldots$ ) are not Lojasiewicz numbers.

The two results give rise to the following problem:

Problem. Give an effective description of the rationals $N+b / a$ which are Lojasiewicz numbers.

Our first result is

Main Theorem 1. All the numbers in (3) satisfying

$$
a+b \leq N
$$

and the number 1 are Lojasiewicz numbers. Moreover, these numbers are exactly the Eojasiewicz exponents of singularities nondegenerate in Kouchnirenko's sense [K].

The rationals

$$
N+\frac{b}{a}, \quad a, b, N \in \mathbb{Z}, 0 \leq b<a<N, a+b \leq N,
$$

and the number 1 will be called regular Łojasiewicz numbers. The remaining Łojasiewicz numbers will be called nonregular. Thus $\mathcal{L}_{0}(f)$ is nonregular if and only if

$$
\mathcal{L}_{0}(f)=N+\frac{b}{a}, \quad 0<b<a<N, \operatorname{GCD}(a, b)=1, a+b>N .
$$

The problem of characterizing nonregular Łojasiewicz numbers (or at least finding some of them) is much more difficult. The following theorem gives necessary conditions for a rational number to be a nonregular Łojasiewicz number.

MAIN TheOREM 2. If a rational number $\lambda=N+b / a$ is a nonregular Eojasiewicz number then

(i) a is a composite number strictly greater than 8 ,

(ii) $a+6<\lambda<2 a-1$.

Clearly the above theorem implies (4) which is the main result in [Ga-P]. Moreover, we will prove the following result on the existence of nonregular Łojasiewicz numbers. 
Main Theorem 3. For every composite number $a>8$ there exists a nonregular Eojasiewicz number with the smallest denominator equal to a.

We will see in Section 7 that $15 \frac{8}{9}$ is the smallest nonregular Eojasiewicz number. Theorems 2 and 3 imply that the set of nonregular Łojasiewicz numbers is infinite but the set of such numbers with fixed denominator is finite.

To prove our main results we will study singularities whose Eojasiewicz exponent has large denominator, i.e. plane curve singularities $(C, 0)$ with the Łojasiewicz exponent of the form $N+b / a, a, b$ coprime and $a>\frac{1}{2} \mathrm{~m}(C)$ where $\mathrm{m}(C)$ stands for the multiplicity of the germ $(C, 0)$. This condition imposes rather strong restrictions on the equisingularity class of $(C, 0)$ (Section 3$)$.

The paper is organized as follows. In Section 2 we collect auxiliary results concerning the characteristic of branches, the intersection multiplicity of branches and the Łojasiewicz exponents of holomorphic functions. In Section 3 we investigate the Łojasiewicz numbers with large denominators. In Section 4 we construct singularities with given Łojasiewicz numbers. Section 5 is devoted to arithmetical results needed in the proofs of the Main Theorems. In Section 6 we give proofs of the main results. In Section 7 we give remarks and examples.

2. Auxiliary results. We need some auxiliary notions coming from the theory of plane curve branches (see [Z2, pp. 7-25]). A sequence of strictly positive integers $\beta_{0}, \ldots, \beta_{g}$ is called a characteristic sequence if the following conditions hold:

$$
\beta_{i}<\beta_{i+1} \quad \text { for } i \in\{0,1, \ldots, g-1\}
$$

and if we put $e_{i}=\operatorname{GCD}\left(\beta_{0}, \ldots, \beta_{i}\right)$ then

$$
e_{i}>e_{i+1} \quad \text { for } i \in\{0,1, \ldots, g-1\} \quad \text { and } \quad e_{g}=1 .
$$

For every characteristic sequence $\beta_{0}, \ldots, \beta_{g}$ we define the derived characteristic sequence $\bar{\beta}_{0}, \ldots, \bar{\beta}_{g}$ by setting

$$
\bar{\beta}_{0}=\beta_{0}, \quad \bar{\beta}_{1}=\beta_{1}, \quad \bar{\beta}_{i}=\beta_{i}+\frac{1}{e_{i-1}} \sum_{j=1}^{i-1}\left(e_{j-1}-e_{j}\right) \beta_{j} \quad \text { for } i \in\{2, \ldots, g\} .
$$

It is easy to check the following properties:

$$
\begin{aligned}
e_{i-1} \bar{\beta}_{i}<e_{i} \bar{\beta}_{i+1} & \text { for } i \in\{1, \ldots, g-1\}, \\
\operatorname{GCD}\left(\bar{\beta}_{0}, \ldots, \bar{\beta}_{i}\right)=e_{i} & \text { for } i \in\{0, \ldots, g-1\} .
\end{aligned}
$$

The semigroup $\left\langle\bar{\beta}_{0}, \ldots, \bar{\beta}_{g}\right\rangle=\mathbb{N} \bar{\beta}_{0}+\cdots+\mathbb{N} \bar{\beta}_{g}$ plays an important role in the theory of branches (see [Z2]). Every element of $\left\langle\bar{\beta}_{0}, \ldots, \bar{\beta}_{g}\right\rangle$ has a 
unique representation in the form $\sum_{i=0}^{g} a_{i-1} \bar{\beta}_{i}$ where $a_{-1}, a_{0}, \ldots, a_{g-1}$ are nonnegative integers such that $a_{i}<e_{i} / e_{i+1}$ for $i \geq 0$ (no restriction on $a_{-1}$ !).

Throughout the paper we use standard notation: the intersection multiplicity of plane curve germs $(C, 0)$ and $(D, 0)$ is denoted by $(C, D)_{0}$. The semigroup $\Gamma(C, 0)$ of a plane branch $(C, 0)$ is generated by the intersection numbers $(C, D)_{0}$ where $(D, 0)$ runs over all plane curve germs whose components are different from $(C, 0)$.

Let $(C, 0)$ be a plane branch with multiplicity $\mathrm{m}(C)=n>1$. Let $(x, y)$ be a system of coordinates such that the line $x=0$ intersects $(C, 0)$ with multiplicity $n$. Then there is a local equation of $(C, 0)$ of the form $f(x, y)=0$ where $f(x, y)=y^{n}+a_{1}(x) y^{n-1}+\cdots+a_{n}(x) \in \mathbb{C}\{x, y\}, a_{i}(0)=0$, is an irreducible distinguished polynomial. Write $f(x, y)=\prod_{i=1}^{n}\left(y-y_{i}\left(x^{1 / n}\right)\right)$ in $\mathbb{C}\left\{x^{1 / n}\right\}[y]$ and recall that the Puiseux series $y_{1}\left(x^{1 / n}\right), \ldots, y_{n}\left(x^{1 / n}\right)$ form a cycle, i.e. there is a power series $y(t) \in \mathbb{C}\{t\}$ such that $y_{i}\left(x^{1 / n}\right)=y\left(\varepsilon^{i} x^{1 / n}\right)$ where $\varepsilon$ is a primitive root of unity of degree $n$. There exists a unique characteristic sequence $\beta_{0}, \ldots, \beta_{g}$ such that

$$
\left\{\operatorname{ord}\left(y_{i}\left(x^{1 / n}\right)-y_{j}\left(x^{1 / n}\right)\right): i \neq j\right\}=\left\{\beta_{1} / \beta_{0}, \ldots, \beta_{g} / \beta_{0}\right\}
$$

and $\beta_{0}=n$. We call $\left(\beta_{0}, \ldots, \beta_{g}\right)$ the characteristic of $(C, 0)$. The derived characteristic sequence $\left(\bar{\beta}_{0}, \ldots, \bar{\beta}_{g}\right)$ generates the semigroup $\Gamma(C, 0)$ of the branch $(C, 0)$. Recall that $g$ is the number of characteristic pairs of $(C, 0)$. Every characteristic sequence is equal to the characteristic of a branch. By convention we put $\beta_{0}=1$ for every smooth branch.

Let $(D, 0)$ be another branch with multiplicity $\mathrm{m}(D)=n^{\prime}>1$ such that the line $x=0$ intersects $(D, 0)$ with multiplicity $n^{\prime}$. Let $g(x, y)=$ $\prod_{j=1}^{n^{\prime}}\left(y-z_{j}\left(x^{1 / n^{\prime}}\right)\right) \in \mathbb{C}\left\{x^{1 / n^{\prime}}\right\}[y]$ be an irreducible power series such that $g(x, y)=0$ is a local equation of $(D, 0)$.

We denote by $\operatorname{Char}(C, 0)$ the characteristic of the branch $(C, 0)$. The order of contact of the branches $(C, 0)$ and $(D, 0)$ is defined by

$$
\operatorname{cont}(C, D)=\max _{i, j}\left\{\operatorname{ord}\left(y_{i}\left(x^{1 / n}\right)-z_{j}\left(x^{1 / n^{\prime}}\right)\right\} .\right.
$$

If $(C, 0) \neq(D, 0)$ then $\operatorname{cont}(C, D)<\infty$ is a rational number $\geq 1$. Clearly $\operatorname{cont}(C, D)=\operatorname{cont}(D, C)$ and for each branch $(E, 0)$,

$$
\operatorname{cont}(C, D) \geq \inf \{\operatorname{cont}(C, E), \operatorname{cont}(E, D)\}
$$

with equality if $\operatorname{cont}(C, E) \neq \operatorname{cont}(E, D)$. According to Chądzyński and Krasiński ([Cha-Kra, Theorem 4]) $\operatorname{cont}(C, D)$ is equal to the best separation exponent of the pair $(C, 0)$ and $(D, 0)$.

Let us recall

SMITH-ZARISKI FORMULA FOR THE INTERSECTION MULTIPLICITY (see [S] and [Z1, pp. 927-931]). Let $(C, 0)$ be a branch of characteristic $\left(\beta_{0}, \ldots, \beta_{g}\right)$ 
and let $(D, 0) \neq(C, 0)$ be another branch. Let $k>0$ be the smallest integer such that $\operatorname{cont}(C, D) \leq \beta_{k} / \beta_{0}$ (by definition $\left.\beta_{g+1} / \beta_{0}=+\infty\right)$. Then

$$
\frac{(C, D)_{0}}{\mathrm{~m}(D)}=\sum_{i=1}^{k-1}\left(e_{i-1}-e_{i}\right) \frac{\beta_{i}}{\beta_{0}}+e_{k-1} \operatorname{cont}(C, D) .
$$

Moreover if $\left(\beta_{0}^{\prime}, \ldots, \beta_{g^{\prime}}^{\prime}\right)$ is the characteristic of $(D, 0)$ then $k \leq g^{\prime}+1$, $\operatorname{cont}(C, D) \leq \beta_{k}^{\prime} / \beta_{0}^{\prime}$ and $\beta_{i} / \beta_{0}=\beta_{i}^{\prime} / \beta_{0}^{\prime}$ for $i<k$.

REMARK 2.1. The equalities $\beta_{i} / \beta_{0}=\beta_{i}^{\prime} / \beta_{0}^{\prime}$ for $i<k$ imply $e_{i} / e_{0}=e_{i}^{\prime} / e_{0}^{\prime}$ for $i<k$ and consequently the Smith-Zariski formula can be rewritten in the form

$$
\frac{(C, D)_{0}}{\mathrm{~m}(C)}=\sum_{i=1}^{k-1}\left(e_{i-1}^{\prime}-e_{i}^{\prime}\right) \frac{\beta_{i}^{\prime}}{\beta_{0}^{\prime}}+e_{k-1}^{\prime} \operatorname{cont}(C, D) .
$$

REMARK 2.2. Let $k>0$ be an integer. Then $\operatorname{cont}(C, D) \leq \beta_{k} / \beta_{0}$ if and only if $(C, D)_{0} / \mathrm{m}(D) \leq e_{k-1} \bar{\beta}_{k} / \beta_{0}$. One has $\operatorname{cont}(C, D)=\beta_{k} / \beta_{0}$ if and only if $(C, D)_{0} / \mathrm{m}(D)=e_{k-1} \bar{\beta}_{k} / \beta_{0}$ (see [Gw-P, Lemma 3.4]).

REMARK 2.3. Let $(C, 0)$ be a branch of characteristic $\left(\beta_{0}, \ldots, \beta_{g}\right)$. Then for every $0<k \leq g$ there is a branch $(D, 0)$ such that $(C, D)_{0}=\bar{\beta}_{k}$ and $\mathrm{m}(D)=\beta_{0} / e_{k-1}($ see $[\mathrm{Gw}-\mathrm{P}$, Lemma 3.1]).

The following statement can be deduced from the corresponding property of $\operatorname{cont}(C, D)$ by using the Smith-Zariski formula (see also [Ch-P]).

Strong Triangle Inequality. If $(C, 0),(D, 0)$ and $(E, 0)$ are branches, then

$$
\frac{(C, D)_{0}}{\mathrm{~m}(C) \mathrm{m}(D)} \geq \inf \left\{\frac{(C, E)_{0}}{\mathrm{~m}(C) \mathrm{m}(E)}, \frac{(E, D)_{0}}{\mathrm{~m}(E) \mathrm{m}(D)}\right\},
$$

with equality if the two quotients on the right hand side are different.

For every branch $(C, 0)$ with characteristic $\left(\beta_{0}, \ldots, \beta_{g}\right), g>0$, we put

$$
\eta(C)=\frac{e_{g-1} \bar{\beta}_{g}}{\beta_{0}}
$$

(it is the greatest polar invariant of $(C, 0)$, see for example [P2]). If $(C, 0)$ is smooth then $\eta(C)=-\infty$. If $\operatorname{Char}(C, 0)=\operatorname{Char}(D, 0)$ then we write $(C, 0) \cong(D, 0)$ and call the branches $(C, 0)$ and $(D, 0)$ equisingular.

Proposition 2.4. Let $(C, 0)$ and $(D, 0)$ be two branches. Suppose that $\operatorname{Char}(C, 0)=\left(\beta_{0}, \ldots, \beta_{g}\right)$.

(i) If

$$
\frac{(C, D)_{0}}{\mathrm{~m}(D)}>\eta(C) \quad \text { and } \quad \frac{(C, D)_{0}}{\mathrm{~m}(C)}>\eta(D)
$$

then $(C, 0) \cong(D, 0)$. 
(ii) If

$$
\frac{(C, D)_{0}}{\mathrm{~m}(C)}=\eta(D) \quad \text { and } \quad \frac{(C, D)_{0}}{\mathrm{~m}(D)}=\eta(C),
$$

then $(C, 0) \cong(D, 0)$.

(iii) If

$$
\frac{(C, D)_{0}}{\mathrm{~m}(C)}>\eta(D) \quad \text { and } \quad \frac{(C, D)_{0}}{\mathrm{~m}(D)}=\eta(C)
$$

then $\operatorname{Char}(D, 0)=\left(\beta_{0} / e_{g-1}, \ldots, \beta_{g-1} / e_{g-1}\right)$ and $(C, D)_{0}=\bar{\beta}_{g}$.

(iv) If $(C, D)_{0} / \mathrm{m}(D)<\eta(C)$ then $\left.(C, D)_{0} \equiv 0\left(\bmod e_{g-1}\right)\right)$.

Proof. (i) We may assume that $(C, 0)$ and $(D, 0)$ are singular. Let

$$
\operatorname{Char}(D, 0)=\left(\beta_{0}^{\prime}, \ldots, \beta_{g^{\prime}}^{\prime}\right) \text {. }
$$

By Remark 2.2 we get $\operatorname{cont}(C, D)>\beta_{g} / \beta_{0}$ and $\operatorname{cont}(C, D)>\beta_{g^{\prime}}^{\prime} / \beta_{0}^{\prime}$. By the Smith-Zariski formula $g^{\prime}=g$ and $\beta_{i} / \beta_{0}=\beta_{i}^{\prime} / \beta_{0}^{\prime}$ for $i \in\{1, \ldots, g\}$. By Remark $2.2, e_{i} / e_{0}=e_{i}^{\prime} / e_{0}^{\prime}$ for $i \in\{1, \ldots, g\}$ and consequently $\left(\beta_{0}, \ldots, \beta_{g}\right)=$ $\left(\beta_{0}^{\prime}, \ldots, \beta_{g}^{\prime}\right)$.

(ii) We get $\operatorname{cont}(C, D)=\beta_{g} / \beta_{0}=\beta_{g^{\prime}}^{\prime} / \beta_{0}^{\prime}$ by Remark 2.2. By the SmithZariski formula $g=g^{\prime}$ and $\beta_{i} / \beta_{0}=\beta_{i}^{\prime} / \beta_{0}^{\prime}$ for $i<g$. Thus $\left(\beta_{0}, \ldots, \beta_{g}\right)=$ $\left(\beta_{0}^{\prime}, \ldots, \beta_{g}^{\prime}\right)$.

(iii) Using Remark 2.2 we get $\operatorname{cont}(C, D)=\beta_{g} / \beta_{0}$ and $\operatorname{cont}(C, D)$ $>\beta_{g^{\prime}}^{\prime} / \beta_{0}^{\prime}$. Thus $g^{\prime}=g-1$ and $\beta_{k} / \beta_{0}=\beta_{k}^{\prime} / \beta_{0}^{\prime}$ for $k \in\{1, \ldots, g-1\}$. Consequently, $e_{g-1} / \beta_{0}=e_{g^{\prime}}^{\prime} / \beta_{0}^{\prime}\left(e_{g^{\prime}}^{\prime}=1\right)$ and $\beta_{0}^{\prime}=\beta_{0} / e_{g-1}$, whence $\left(\beta_{0}^{\prime}, \ldots, \beta_{g^{\prime}}^{\prime}\right)$ $=\left(\beta_{0} / e_{g-1}, \ldots, \beta_{g-1} / e_{g-1}\right)$. Now, we get

$$
(C, D)_{0}=\eta(C) \mathrm{m}(C)=\left(\frac{e_{g-1} \bar{\beta}_{g}}{\beta_{0}}\right)\left(\frac{\beta_{0}}{e_{g-1}}\right)=\bar{\beta}_{g} .
$$

(iv) Let $\left(D_{1}, 0\right)$ be a branch such that

$$
\operatorname{Char}\left(D_{1}, 0\right)=\left(\beta_{0} / e_{g-1}, \ldots, \beta_{g-1} / e_{g-1}\right) \quad \text { and } \quad\left(C, D_{1}\right)_{0}=\bar{\beta}_{g} \text {. }
$$

Then $\left(C, D_{1}\right)_{0} / \mathrm{m}\left(D_{1}\right)=\eta(C)$ and $\left(C, D_{1}\right)_{0} / \mathrm{m}(C)>\eta\left(D_{1}\right)$. Consider the sequence

One has

$$
\frac{(C, D)_{0}}{\mathrm{~m}(C) \mathrm{m}(D)}, \quad \frac{\left(C, D_{1}\right)_{0}}{\mathrm{~m}(C) \mathrm{m}\left(D_{1}\right)}, \quad \frac{\left(D, D_{1}\right)_{0}}{\mathrm{~m}(D) \mathrm{m}\left(D_{1}\right)} .
$$

$$
\frac{(C, D)_{0}}{\mathrm{~m}(C) \mathrm{m}(D)}<\frac{\eta(C)}{\mathrm{m}(C)}=\frac{e_{g-1} \bar{\beta}_{g}}{\beta_{0}^{2}} \quad \text { and } \quad \frac{\left(C, D_{1}\right)_{0}}{\mathrm{~m}(C) \mathrm{m}\left(D_{1}\right)}=\frac{\eta(C)}{\mathrm{m}(C)}=\frac{e_{g-1} \bar{\beta}_{g}}{\beta_{0}^{2}}
$$

Therefore by the Strong Triangle Inequality

$$
\frac{\left(D, D_{1}\right)_{0}}{\mathrm{~m}(D) \mathrm{m}\left(D_{1}\right)}=\frac{(C, D)_{0}}{\mathrm{~m}(C) \mathrm{m}(D)}
$$


and so

$$
(C, D)_{0}=\frac{\mathrm{m}(C)}{\mathrm{m}\left(D_{1}\right)}\left(D, D_{1}\right)_{0}=e_{g-1}\left(D, D_{1}\right)_{0}
$$

Let $(C, 0)$ be a germ with equation $f=0$, where $f$ is a holomorphic function with an isolated critical point at 0 . Let $(C, 0)=\bigcup_{i=1}^{r}\left(C_{i}, 0\right)$ be the decomposition of $(C, 0)$ into irreducible components. Then we have

Formula FOR THE ŁoJASIEWICZ ExpOnent (see [P2, Theorem 1.3 and Corollary 1.5]). With the notation introduced above,

$$
\mathcal{L}_{0}(f)+1=\max _{i=1}^{r}\left\{\max \left\{\sup _{j \neq i} \frac{\left(C_{i}, C_{j}\right)_{0}}{\mathrm{~m}\left(C_{j}\right)}, \eta\left(C_{i}\right)\right\}+\frac{1}{\mathrm{~m}\left(C_{i}\right)} \sum_{j \neq i}\left(C_{i}, C_{j}\right)_{0}\right\} .
$$

We will say that a branch $\left(C_{i}, 0\right)$ of $(C, 0)$ is minimal if

$$
\mathcal{L}_{0}(f)+1=\max \left\{\sup _{j \neq i} \frac{\left(C_{i}, C_{j}\right)_{0}}{\mathrm{~m}\left(C_{j}\right)}, \eta\left(C_{i}\right)\right\}+\frac{1}{\mathrm{~m}\left(C_{i}\right)} \sum_{j \neq i}\left(C_{i}, C_{j}\right)_{0} .
$$

Proposition 2.5. Suppose that

$$
\mathcal{L}_{0}(f)+1 \neq \eta\left(C_{i}\right)+\frac{1}{\mathrm{~m}\left(C_{i}\right)} \sum_{j \neq i}\left(C_{i}, C_{j}\right)_{0} \quad \text { for } i \in\{1, \ldots, r\} .
$$

Then for every minimal branch $\left(C_{i}, 0\right)$ there is a branch $\left(C_{j}, 0\right)$ with $j \neq i$ such that $\left(C_{j}, 0\right) \cong\left(C_{i}, 0\right)$ and

$$
\mathcal{L}_{0}(f)+1=\frac{\left(C_{i}, C_{j}\right)_{0}}{\mathrm{~m}\left(C_{j}\right)}+\sum_{k \neq i} \frac{\left(C_{i}, C_{k}\right)_{0}}{\mathrm{~m}\left(C_{i}\right)} .
$$

Proof. Let $\left(C_{i}, 0\right)$ be a minimal branch of $(C, 0)$. Then by the formula for the Łojasiewicz exponent,

$$
\mathcal{L}_{0}(f)+1=\frac{\left(C_{i}, C_{j}\right)_{0}}{\mathrm{~m}\left(C_{j}\right)}+\sum_{k \neq i} \frac{\left(C_{i}, C_{k}\right)_{0}}{\mathrm{~m}\left(C_{i}\right)}
$$

for some $j$. We will show that $\left(C_{j}, 0\right) \cong\left(C_{i}, 0\right)$. We may assume that $i=1$, $j=2$, that is,

$$
\mathcal{L}_{0}(f)+1=\frac{\left(C_{1}, C_{2}\right)_{0}}{\mathrm{~m}\left(C_{2}\right)}+\frac{1}{\mathrm{~m}\left(C_{1}\right)} \sum_{j \neq 1}\left(C_{1}, C_{j}\right) .
$$

By the assumption we get

$$
\mathcal{L}_{0}(f)+1>\eta\left(C_{1}\right)+\frac{1}{\mathrm{~m}\left(C_{1}\right)} \sum_{j \neq 1}\left(C_{1}, C_{j}\right) .
$$

Thus, by (9) and (10) we get

$$
\frac{\left(C_{1}, C_{2}\right)_{0}}{\mathrm{~m}\left(C_{2}\right)}>\eta\left(C_{1}\right)
$$


Using again the assumption of the proposition, we obtain

$$
\mathcal{L}_{0}(f)+1>\eta\left(C_{2}\right)+\frac{1}{\mathrm{~m}\left(C_{2}\right)} \sum_{j \neq 2}\left(C_{2}, C_{j}\right)
$$

and a simple calculation based on (9) and (12) gives

$$
\frac{\left(C_{1}, C_{2}\right)_{0}}{\mathrm{~m}\left(C_{1}\right)}>\eta\left(C_{2}\right)+\sum_{j \neq 1,2}\left(\frac{\left(C_{2}, C_{j}\right)_{0}}{\mathrm{~m}\left(C_{2}\right) \mathrm{m}\left(C_{j}\right)}-\frac{\left(C_{1}, C_{j}\right)_{0}}{\mathrm{~m}\left(C_{1}\right) \mathrm{m}\left(C_{j}\right)}\right) \mathrm{m}\left(C_{j}\right) .
$$

Note that

$$
\frac{\left(C_{2}, C_{j}\right)_{0}}{\mathrm{~m}\left(C_{2}\right) \mathrm{m}\left(C_{j}\right)} \geq \frac{\left(C_{1}, C_{j}\right)_{0}}{\mathrm{~m}\left(C_{1}\right) \mathrm{m}\left(C_{j}\right)} \quad \text { for } j \neq 1,2
$$

by the Strong Triangle Inequality, for $\frac{\left(C_{1}, C_{j}\right)_{0}}{\mathrm{~m}\left(C_{1}\right) \mathrm{m}\left(C_{j}\right)} \leq \frac{\left(C_{1}, C_{2}\right)_{0}}{\mathrm{~m}\left(C_{1}\right) \mathrm{m}\left(C_{2}\right)}$.

Now (13) and (14) imply

$$
\frac{\left(C_{1}, C_{2}\right)_{0}}{\mathrm{~m}\left(C_{1}\right)}>\eta\left(C_{2}\right)
$$

Using (11), (15) and Proposition 2.4 we get $\left(C_{1}, 0\right) \cong\left(C_{2}, 0\right)$.

COROLlary 2.6. With the assumption and notation of Proposition 2.5 we have

(i) $\mathcal{L}_{0}(f)+1=\left(\left(C_{i}, C_{j}\right)_{0}+\sum_{k \neq i}\left(C_{i}, C_{k}\right)\right) / \mathrm{m}\left(C_{j}\right)$ for some $j \neq i$ with $\mathrm{m}\left(C_{j}\right)=\mathrm{m}\left(C_{i}\right)$.

(ii) $\mathcal{L}_{0}(f)+1$ has the (minimal) denominator less than or equal to $\frac{1}{2} \mathrm{~m}(C)$.

Proof. The first property follows immediately from Proposition 2.5, for $\left(C_{i}, 0\right) \cong\left(C_{j}, 0\right)$ implies $\mathrm{m}\left(C_{i}\right)=\mathrm{m}\left(C_{j}\right)$. The denominator of $\mathcal{L}_{0}(f)+1$ is less than or equal to $\mathrm{m}\left(C_{j}\right) \leq \frac{1}{2} \mathrm{~m}(C)$ since $2 \mathrm{~m}\left(C_{j}\right)=\mathrm{m}\left(C_{i}\right)+\mathrm{m}\left(C_{j}\right) \leq \mathrm{m}(C)$.

Proposition 2.7. Suppose that

$$
\mathcal{L}_{0}(f)+1=\eta\left(C_{i}\right)+\frac{1}{\mathrm{~m}\left(C_{i}\right)} \sum_{j \neq i}\left(C_{i}, C_{j}\right)_{0} \quad \text { for some } i \in\{1, \ldots, r\} .
$$

Then

$$
\sup _{j \neq i} \frac{\left(C_{i}, C_{j}\right)_{0}}{\mathrm{~m}\left(C_{j}\right)} \leq \eta\left(C_{i}\right) .
$$

If the inequality is strict then the denominator of $\mathcal{L}_{0}(f)+1$ is less than or equal to $\frac{1}{2} \mathrm{~m}(C)$.

Proof. The inequality in the statement follows from the formula for the Łojasiewicz exponent. Let $\left(\beta_{0}, \ldots, \beta_{g}\right)$ be the characteristic of $\left(C_{i}, 0\right)$. Suppose that the inequality in the statement is strict. Then by Proposition 2.4 
we get $\left(C_{i}, C_{j}\right)_{0} \equiv 0\left(\bmod e_{g-1}\right)$ for all $j \neq i$. Consequently, we see that the fraction

$$
\mathcal{L}_{0}(f)+1=\frac{\mathrm{m}\left(C_{i}\right) \eta\left(C_{i}\right)+\sum_{j \neq i}\left(C_{i}, C_{j}\right)_{0}}{\mathrm{~m}\left(C_{i}\right)}=\frac{e_{g-1} \bar{\beta}_{g}+\left(\text { a multiple of } e_{g-1}\right)}{\beta_{0}}
$$

has the denominator $\leq \beta_{0} / e_{g-1} \leq \mathrm{m}\left(C_{i}\right) / 2 \leq \mathrm{m}(C) / 2$.

To illustrate the above propositions we prove

Proposition 2.8. Let $(C, 0)=\bigcup_{i=1}^{r}\left(C_{i}, 0\right)$ be a decomposition of the germ $(C, 0)$ with local equation $f=0$ into branches. Then there exist a branch $\left(C_{i_{0}}, 0\right)$ and an integer $\gamma \in \Gamma\left(C_{i_{0}}, 0\right)$ such that

$$
\mathcal{L}_{0}(f)+1=\frac{\gamma}{\mathrm{m}\left(C_{i_{0}}\right)} .
$$

Proof. If the assumption of Proposition 2.5 holds then the assertion follows from Corollary 2.6. Otherwise use Proposition 2.7.

3. Łojasiewicz numbers with large denominators. Let $f$ with $f(0)=0$ be a holomorphic function near $0 \in \mathbb{C}^{2}$ with an isolated critical point at 0 and let $(C, 0)$ be a germ with local equation $f=0$. Suppose that $\mathcal{L}_{0}(f)$ is not an integer and write $\mathcal{L}_{0}(f)=N+b / a$ with $0<b<a$ and $\operatorname{GCD}(a, b)=1$. From Proposition 2.8 it follows that $a \leq \mathrm{m}(C)$.

Lemma 3.1. If $\mathcal{L}_{0}(f)=N+b / a$ with $a \leq \frac{1}{2} \mathrm{~m}(C)$ then $\mathcal{L}_{0}(f)$ is a regular Łojasiewicz number.

Proof. From $a \leq \frac{1}{2} \mathrm{~m}(C)$ we get $a+b<2 a \leq \mathrm{m}(C)$ and hence $a+b \leq$ $\mathrm{m}(C)-1$. On the other hand, it is easy to check that $\mathcal{L}_{0}(f) \geq \mathrm{m}(C)-1$, which implies $N \geq \mathrm{m}(C)-1 \geq a+b$.

By Lemma 3.1 when looking for the singularities $f=0$ with nonregular $\mathcal{L}_{0}(f)$ we may restrict our attention to those for which $\mathcal{L}_{0}(f)=N+b / a$, $0<b<a, \operatorname{GCD}(a, b)=1$ and $a>\frac{1}{2} \mathrm{~m}(C)$. In this case we say that $\mathcal{L}_{0}(f)$ has large denominator.

ThEOREM 3.2. Suppose that the Eojasiewicz number $\mathcal{L}_{0}(f)$ of a plane curve singularity $(C, 0)$ has large denominator. Then the germ $(C, 0)$ has at least two branches and there is a decomposition $(C, 0)=\bigcup_{i=1}^{r}\left(C_{i}, 0\right)$ into branches such that the following conditions are fulfilled:

(i) The branch $\left(C_{1}, 0\right)$ is singular. If $\left(\beta_{0}, \ldots, \beta_{g}\right)$ is the characteristic of $\left(C_{1}, 0\right)$ then $\left(\beta_{0} / e_{g-1}, \ldots, \beta_{g-1} / e_{g-1}\right)$ is the characteristic of $\left(C_{2}, 0\right)$. Moreover $\left(C_{1}, C_{2}\right)_{0}=\bar{\beta}_{g}$. 
(ii) For every $i \neq 1,2$,

$$
\frac{\left(C_{1}, C_{i}\right)_{0}}{\mathrm{~m}\left(C_{1}\right) \mathrm{m}\left(C_{i}\right)}=\frac{\left(C_{2}, C_{i}\right)_{0}}{\mathrm{~m}\left(C_{2}\right) \mathrm{m}\left(C_{i}\right)}<\frac{\left(C_{1}, C_{2}\right)_{0}}{\mathrm{~m}\left(C_{1}\right) \mathrm{m}\left(C_{2}\right)} .
$$

(iii) $\mathcal{L}_{0}(f)+1=\left(e_{g-1} \bar{\beta}_{g}+\bar{\beta}_{g}+\delta\right) / \beta_{0}$ where $\delta=\sum_{i \neq 1,2}\left(C_{1}, C_{i}\right)_{0}$. Moreover $\delta \in\left\langle\bar{\beta}_{0}, \ldots, \bar{\beta}_{g-1}\right\rangle$.

Let $\left(\beta_{0}, \ldots, \beta_{g}\right)$ be a characteristic sequence. We say that a rational number $\lambda$ is associated with $\left(\beta_{0}, \ldots, \beta_{g}\right)$ if there is an integer $\delta \in\left\langle\bar{\beta}_{0}, \ldots, \bar{\beta}_{g-1}\right\rangle$ such that

$$
\lambda+1=\frac{e_{g-1} \bar{\beta}_{g}+\bar{\beta}_{g}+\delta}{\beta_{0}} .
$$

Using Theorem 3.2 we get

Corollary 3.3. If the Eojasiewicz number $\mathcal{L}_{0}(f)$ has large denominator then there is a singular branch $\left(C_{1}, 0\right)$ of $(C, 0)$ such that $\mathcal{L}_{0}(f)$ is associated with the characteristic of $\left(C_{1}, 0\right)$.

Another application of Theorem 3.2 is the following

COROLlARY 3.4. If the Eojasiewicz number $\mathcal{L}_{0}(f)$ has large denominator then there is a decomposition $(C, 0)=\bigcup_{i=1}^{r}\left(C_{i}, 0\right)$ into branches such that $\sum_{i=2}^{r} \mathrm{~m}\left(C_{i}\right)<\mathrm{m}\left(C_{1}\right)$ and $\mathrm{m}\left(C_{2}\right)$ divides $\mathrm{m}\left(C_{1}\right)$.

Proof. By Theorem 3.2 there is a decomposition $(C, 0)=\bigcup_{i=1}^{r}\left(C_{i}, 0\right)$ into branches such that $\left(C_{1}, 0\right)$ is singular and $\mathcal{L}_{0}(f)$ is associated with the characteristic $\left(\beta_{0}, \ldots, \beta_{g}\right)$ of $\left(C_{1}, 0\right)$. It is easy to see that $\beta_{0}=\mathrm{m}\left(C_{1}\right)$ is the denominator of $\mathcal{L}_{0}(f)$. Therefore $\mathrm{m}\left(C_{1}\right)>\frac{1}{2} \mathrm{~m}(C)$ and the corollary follows.

Proof of Theorem 3.2. By Propositions 2.4 and 2.7 we may assume that there is a decomposition $(C, 0)=\bigcup_{i=1}^{r}\left(C_{i}, 0\right), r \geq 2$, such that

$$
\begin{gathered}
\mathcal{L}_{0}(f)+1=\eta\left(C_{1}\right)+\frac{1}{\mathrm{~m}\left(C_{1}\right)} \sum_{j \neq 1}\left(C_{1}, C_{j}\right)_{0}, \\
\max _{j \neq 1} \frac{\left(C_{1}, C_{j}\right)_{0}}{\mathrm{~m}\left(C_{j}\right)}=\frac{\left(C_{1}, C_{2}\right)_{0}}{\mathrm{~m}\left(C_{2}\right)}=\eta\left(C_{1}\right) .
\end{gathered}
$$

Using (16) and the formula for the Eojasiewicz exponent, we get

$$
\begin{aligned}
\eta\left(C_{1}\right)+\frac{1}{\mathrm{~m}\left(C_{1}\right)} & \sum_{j \neq 1}\left(C_{1}, C_{j}\right)_{0}=\mathcal{L}_{0}(f)+1 \\
& \geq \max \left\{\max _{j \neq 2} \frac{\left(C_{2}, C_{j}\right)_{0}}{\mathrm{~m}\left(C_{j}\right)}, \eta\left(C_{2}\right)\right\}+\frac{1}{\mathrm{~m}\left(C_{2}\right)} \sum_{j \neq 2}\left(C_{2}, C_{j}\right)_{0},
\end{aligned}
$$


and by (17),

$$
\begin{aligned}
\frac{\left(C_{1}, C_{2}\right)_{0}}{\mathrm{~m}\left(C_{1}\right)} \geq & \max \left\{\max _{j \neq 2} \frac{\left(C_{2}, C_{j}\right)_{0}}{\mathrm{~m}\left(C_{j}\right)}, \eta\left(C_{2}\right)\right\} \\
& +\sum_{j \neq 1,2}\left(\frac{\left(C_{2}, C_{j}\right)_{0}}{\mathrm{~m}\left(C_{2}\right) \mathrm{m}\left(C_{j}\right)}-\frac{\left(C_{1}, C_{j}\right)_{0}}{\mathrm{~m}\left(C_{1}\right) \mathrm{m}\left(C_{j}\right)}\right) \mathrm{m}\left(C_{j}\right) .
\end{aligned}
$$

Using (17) and the Strong Triangle Inequality we check that the sum on the right side of the above inequality is positive.

Therefore, we have

$$
\frac{\left(C_{1}, C_{2}\right)_{0}}{\mathrm{~m}\left(C_{1}\right)} \geq \eta\left(C_{2}\right)
$$

and

$$
\frac{\left(C_{1}, C_{2}\right)_{0}}{\mathrm{~m}\left(C_{1}\right)} \geq \max _{j \neq 2} \frac{\left(C_{2}, C_{j}\right)_{0}}{\mathrm{~m}\left(C_{j}\right)} .
$$

We claim that

$$
\frac{\left(C_{1}, C_{2}\right)_{0}}{\mathrm{~m}\left(C_{1}\right)}>\eta\left(C_{2}\right)
$$

In fact, if we had $\left(C_{1}, C_{2}\right)_{0} / \mathrm{m}\left(C_{1}\right)=\eta\left(C_{2}\right)$ then by Proposition 2.4 we would get $\left(C_{1}, 0\right) \cong\left(C_{2}, 0\right)$ and consequently $\mathrm{m}\left(C_{1}\right)=\mathrm{m}\left(C_{2}\right)$, which is impossible because $\mathcal{L}_{0}(f)$ has large denominator. Now, from (17) and (20), by Proposition 2.4 we get $\operatorname{Char}\left(C_{1}, 0\right)=\left(\beta_{0}, \ldots, \beta_{g}\right), \operatorname{Char}\left(C_{2}, 0\right)=\left(\beta_{0} / e_{g-1}, \ldots\right.$, $\left.\beta_{g-1} / e_{g-1}\right)$ and $\left(C_{1}, C_{2}\right)_{0}=\bar{\beta}_{g}$.

Now, we see that (19) can be rewritten in the form

$$
\max _{j \neq 2} \frac{\left(C_{2}, C_{j}\right)_{0}}{\mathrm{~m}\left(C_{j}\right)} \leq \frac{\bar{\beta}_{g}}{\beta_{0}} .
$$

We claim that

$$
\frac{\left(C_{1}, C_{j}\right)_{0}}{\mathrm{~m}\left(C_{j}\right)}<\frac{\left(C_{1}, C_{2}\right)_{0}}{\mathrm{~m}\left(C_{2}\right)} \quad \text { for } j \neq 1,2 .
$$

To check (22) we suppose that there is a $j \neq 1,2$ for which the inequality is not true. Suppose that $j=3$. Thus we may replace $\left(C_{2}, 0\right)$ by $\left(C_{3}, 0\right)$ in the reasoning above to get $\operatorname{Char}\left(C_{3}, 0\right)=\left(\beta_{0} / e_{g-1}, \ldots, \beta_{g-1} / e_{g-1}\right)$ and $\left(C_{1}, C_{3}\right)_{0}=\bar{\beta}_{g}$. By the Strong Triangle Inequality we get

$$
\begin{aligned}
\left(C_{2}, C_{3}\right)_{0} & \geq\left(\frac{\beta_{0}}{e_{g-1}}\right)^{2} \inf \left\{\frac{\left(C_{1}, C_{2}\right)_{0}}{\mathrm{~m}\left(C_{1}\right) \mathrm{m}\left(C_{2}\right)}, \frac{\left(C_{1}, C_{3}\right)_{0}}{\mathrm{~m}\left(C_{1}\right) \mathrm{m}\left(C_{3}\right)}\right\} \\
& =\left(\frac{\beta_{0}}{e_{g-1}}\right)^{2} \frac{e_{g-1} \bar{\beta}_{g}}{\beta_{0}^{2}}=\frac{\bar{\beta}_{g}}{e_{g-1}} .
\end{aligned}
$$


Since $\bar{\beta}_{g} / e_{g-1}$ is not an integer, $\left(C_{2}, C_{3}\right)_{0}>\bar{\beta}_{g} / e_{g-1}$ and $\left(C_{2}, C_{3}\right)_{0} / \mathrm{m}\left(C_{3}\right)>$ $\bar{\beta}_{g} / \beta_{0}$, which contradicts (21). Then (22) and consequently (ii) of Theorem 3.2 hold true.

To prove (iii) observe that

$$
\begin{aligned}
\mathcal{L}_{0}(f)+1 & =\eta\left(C_{1}\right)+\frac{1}{\mathrm{~m}\left(C_{1}\right)} \sum_{j \neq 1}\left(C_{1}, C_{j}\right) \\
& =\frac{\left(C_{1}, C_{2}\right)_{0}}{\mathrm{~m}\left(C_{2}\right)}+\frac{\left(C_{1}, C_{2}\right)_{0}}{\mathrm{~m}\left(C_{1}\right)}+\frac{1}{\mathrm{~m}\left(C_{1}\right)} \sum_{j \neq 1,2}\left(C_{1}, C_{j}\right) \\
& =\frac{e_{g-1} \bar{\beta}_{g}}{\beta_{0}}+\frac{\bar{\beta}_{g}}{\beta_{0}}+\frac{\delta}{\beta_{0}}=\frac{e_{g-1} \bar{\beta}_{g}+\bar{\beta}_{g}+\delta}{\beta_{0}}
\end{aligned}
$$

where $\delta=\sum_{j \neq 1,2}\left(C_{1}, C_{j}\right)_{0}$.

Moreover, we get

$$
\begin{aligned}
\delta & =\sum_{i \neq 1,2}\left(C_{1}, C_{i}\right)_{0}=\sum_{i \neq 1,2} \frac{\mathrm{m}\left(C_{1}\right)}{\mathrm{m}\left(C_{2}\right)}\left(C_{2}, C_{i}\right)_{0} \\
& =e_{g-1} \sum_{i \neq 1,2}\left(C_{2}, C_{i}\right)_{0} \in\left\langle\bar{\beta}_{0}, \ldots, \bar{\beta}_{g-1}\right\rangle
\end{aligned}
$$

for $\Gamma\left(C_{2}, 0\right)=\left\langle\bar{\beta}_{0} / e_{g-1}, \ldots, \bar{\beta}_{g-1} / e_{g-1}\right\rangle$.

EXAMPLE 3.5. From Theorem 3.2 it follows that any Łojasiewicz number $\mathcal{L}_{0}(f)$ with large denominator is associated with a characteristic $\left(\beta_{0}, \ldots, \beta_{g}\right)$, $g \geq 2$, i.e.

$$
\mathcal{L}_{0}(f)+1=\frac{e_{g-1} \bar{\beta}_{g}+\bar{\beta}_{g}+\delta}{\beta_{0}}
$$

for some $\delta \in\left\langle\bar{\beta}_{0}, \ldots, \bar{\beta}_{g-1}\right\rangle$. Unfortunately, this property is not sufficient for a rational number to be a Łojasiewicz number with large denominator. Consider the rational number

$$
\lambda=169 \frac{142}{143} .
$$

It is associated with the characteristic $\left(\beta_{0}, \beta_{1}, \beta_{2}\right)=(143,154,164)$ and $\delta=308$. In fact, we have in this case $\left(e_{0}, e_{1}, e_{2}\right)=(143,11,1),\left(\bar{\beta}_{0}, \bar{\beta}_{1}, \bar{\beta}_{2}\right)=$ $(143,154,2012)$ and

$$
\lambda+1=\frac{e_{g-1} \bar{\beta}_{g}+\bar{\beta}_{g}+\delta}{\beta_{0}} \text { and } \delta=2 \bar{\beta}_{1} \in\left\langle\bar{\beta}_{0}, \bar{\beta}_{1}\right\rangle .
$$

It is easy to check that the characteristic $(143,154,164)$ and $\delta=308$ are unique for $\lambda$.

We claim that $\lambda$ is not the Łojasiewicz number. Assume to the contrary that $\lambda$ is the Łojasiewicz number $\mathcal{L}_{0}(f)$ of a plane curve singularity $(C, 0)$. Since $\lambda$ has large denominator it is associated with a characteristic. By the 
above consideration it is $\left(\beta_{0}, \beta_{1}, \beta_{2}\right)=(143,154,164)$ and $\delta=308$ (determined uniquely). According to Theorem $3.2,(C, 0)$ has a decomposition into branches $(C, 0)=\bigcup_{i=1}^{r}\left(C_{i}, 0\right), r \geq 3$, where

(i) $\left(C_{1}, 0\right)$ has the characteristic $(143,154,164)$,

(ii) $\left(C_{2}, 0\right)$ has the characteristic $(13,14)$,

(iii) $\left(C_{1}, C_{2}\right)=2012,\left(C_{1}, C_{i}\right)=11\left(C_{2}, C_{i}\right)$ for $i \geq 3$,

(iv) $\left(C_{1}, C_{3} \cup \cdots \cup C_{r}\right)=308$.

Since the characteristic of $\left(C_{1}, 0\right)$ is $(143,154,164)$, by (iv) we have two possibilities:

1. $r=4$. Then $C_{3}, C_{4}$ are nonsingular and have the same tangents as $C_{1}$. Hence $\left(C_{3}, C_{4}\right) \geq 2$. By the formula for the Eojasiewicz exponent

where

$$
\mathcal{L}_{0}(f)+1=\max _{i=1}^{4} l_{C_{i}}
$$

$$
l_{C_{i}}=\max \left\{\eta\left(C_{i}\right), \max _{\substack{j=1 \\ j \neq i}}^{4} \frac{\left(C_{i}, C_{j}\right)}{\mathrm{m}\left(C_{j}\right)}\right\}+\frac{1}{\mathrm{~m}\left(C_{i}\right)} \sum_{\substack{j=1 \\ j \neq i}}^{4}\left(C_{i}, C_{j}\right) .
$$

It is easy to check that

$$
l_{C_{3}} \geq 172
$$

which gives a contradiction.

2. $r=3$. Then $\mathrm{m}\left(C_{3}\right)=2$ and $C_{3}$ has the same tangent as $C_{1}$. Hence the characteristic of $C_{3}$ is $\left(\beta_{0}^{\prime}, \beta_{1}^{\prime}\right)=\left(2, \beta_{1}^{\prime}\right)$, where $\beta_{1}^{\prime} \geq 3$. Since we have the general inequality $\eta\left(C_{3}\right) \geq \beta_{1}^{\prime}$, it follows that $\eta\left(C_{3}\right) \geq 3$. Then again by the formula for the Eojasiewicz exponent we easily check that

$$
l_{C_{3}} \geq 171
$$

which gives a contradiction.

4. Singularities with given Łojasiewicz exponent. Let us begin with the following

Proposition 4.1. Let $\left(C_{1}, 0\right)$ be a singular branch with characteristic $\left(\beta_{0}, \ldots, \beta_{g}\right)$ and let $\mathcal{B}=\bigcup_{i=-1}^{g-1} \mathcal{B}_{i}$ be a finite union of finite sets $\mathcal{B}_{i}$ of branches such that:

(i) Every branch of $\mathcal{B}_{-1}$ is smooth and intersects $\left(C_{1}, 0\right)$ with multiplicity $\beta_{0}$. Any two different branches of $\mathcal{B}_{-1}$ intersect with multiplicity 1 .

(ii) For $i \geq 0, \mathcal{B}_{i}$ consists of at most one branch $\left(\Gamma_{i}, 0\right)$. The branch has the characteristic $\left(\beta_{0} / e_{i}, \ldots, \beta_{i} / e_{i}\right)$ and intersects $\left(C_{1}, 0\right)$ with multiplicity $\bar{\beta}_{i+1}$.

(iii) $\mathcal{B}_{g-1} \neq \emptyset$. 
Put $a_{i}=\sharp \mathcal{B}_{i}$ (thus $a_{i}=0$ or $a_{i}=1$ for $0 \leq i<g-2$ and $a_{g-1}=1$; we do not impose any restrictions on $\left.a_{-1}\right)$. Let $f=0$ be the minimal equation of the germ $\left(\bigcup_{i=-1}^{g-1} \mathcal{B}_{i} \cup C_{1}, 0\right)$. Then

$$
\mathcal{L}_{0}(f)+1=\frac{e_{g-1} \bar{\beta}_{g}+\bar{\beta}_{g}+\sum_{i=-1}^{g-2} a_{i} \bar{\beta}_{i+1}}{\beta_{0}} .
$$

By convention $(\Gamma, 0)$, resp. $\left(\Gamma_{-1}, 0\right)$ runs over all branches of $\mathcal{B}$, resp. $\mathcal{B}_{-1}$. To prove Proposition 4.1 we need

Property 4.2. Let $\mathcal{B}_{i} \neq \emptyset$ for some $i \geq 0$. Then, for every $(\Gamma, 0) \in$ $\mathcal{B}-\mathcal{B}_{i}$ :

(i) $\frac{\left(\Gamma_{i}, \Gamma\right)_{0}}{\mathrm{~m}(\Gamma)} \leq \frac{\bar{\beta}_{i+1}}{\beta_{0}}$,

(ii) $\frac{\left(\Gamma_{i}, \Gamma\right)_{0}}{\mathrm{~m}\left(\Gamma_{i}\right)} \leq \frac{\left(C_{1}, \Gamma\right)_{0}}{\mathrm{~m}\left(C_{1}\right)}$.

Proof. Fix $(\Gamma, 0) \in \mathcal{B}-\mathcal{B}_{i}$. Then $(\Gamma, 0) \in \mathcal{B}_{j}$ for a $j \neq i$. We apply the Strong Triangle Inequality to the germs $(\Gamma, 0),\left(\Gamma_{i}, 0\right)$ and $\left(C_{1}, 0\right)$. Since $i \neq j$ we get

$$
\frac{\left(\Gamma, C_{1}\right)_{0}}{\mathrm{~m}(\Gamma) \mathrm{m}\left(C_{1}\right)}=\frac{e_{j} \bar{\beta}_{j+1}}{\beta_{0}^{2}} \neq \frac{e_{i} \bar{\beta}_{i+1}}{\beta_{0}^{2}}=\frac{\left(\Gamma_{i}, C_{1}\right)_{0}}{\mathrm{~m}\left(\Gamma_{i}\right) \mathrm{m}\left(C_{1}\right)}
$$

and consequently

$$
\frac{\left(\Gamma, \Gamma_{i}\right)_{0}}{\mathrm{~m}(\Gamma) \mathrm{m}\left(\Gamma_{i}\right)}=\inf \left\{\frac{e_{i} \bar{\beta}_{i+1}}{\beta_{0}^{2}}, \frac{e_{j} \bar{\beta}_{j+1}}{\beta_{0}^{2}}\right\} .
$$

This implies the assertion.

Proof of Proposition 4.1. Set

$$
l_{1}=\max \left\{\eta\left(C_{1}\right), \max _{\Gamma \in \mathcal{B}} \frac{\left(C_{1}, \Gamma\right)_{0}}{\mathrm{~m}(\Gamma)}\right\}+\frac{1}{\mathrm{~m}\left(C_{1}\right)} \sum_{\Gamma \in \mathcal{B}}\left(C_{1}, \Gamma\right)_{0},
$$

and for every $\left(\Gamma_{i}, 0\right) \in \mathcal{B}_{i}$,

$$
\begin{aligned}
l_{\Gamma_{i}}= & \max \left\{\eta\left(\Gamma_{i}\right), \max _{\Gamma \neq \Gamma_{i}} \frac{\left(\Gamma_{i}, \Gamma\right)_{0}}{\mathrm{~m}(\Gamma)}, \frac{\left(\Gamma_{i}, C_{1}\right)_{0}}{\mathrm{~m}\left(C_{1}\right)}\right\} \\
& +\frac{1}{\mathrm{~m}\left(\Gamma_{i}\right)}\left(\left(\Gamma_{i}, C_{1}\right)_{0}+\sum_{\Gamma \neq \Gamma_{i}}\left(\Gamma_{i}, \Gamma\right)_{0}\right) .
\end{aligned}
$$

By the formula for the Eojasiewicz exponent we get

$$
\mathcal{L}_{0}(f)+1=\max \left\{l_{1}, \max _{i=-1}^{g-1} \max _{\Gamma \in \mathcal{B}_{i}} l_{\Gamma}\right\} .
$$

To calculate $l_{1}$ recall that $\eta\left(C_{1}\right)=e_{g-1} \bar{\beta}_{g} / \beta_{0}$. If $(\Gamma, 0)=\left(\Gamma_{i}, 0\right)$ for an $i \geq 0$ then $\left(C_{1}, \Gamma\right)_{0} / \mathrm{m}(\Gamma)=e_{i} \bar{\beta}_{i+1} / \beta_{0} \leq e_{g-1} \bar{\beta}_{g} / \beta_{0}$, while if $(\Gamma, 0) \in \mathcal{B}_{-1}$ then 
$\left(C_{1}, \Gamma\right)_{0} / \mathrm{m}(\Gamma)=1$, and we get

$$
\max \left\{\eta\left(C_{1}\right), \max _{\Gamma \in \mathcal{B}} \frac{\left(C_{1}, \Gamma\right)_{0}}{\mathrm{~m}(\Gamma)}\right\}=\frac{e_{g-1} \bar{\beta}_{g}}{\beta_{0}}
$$

and consequently

$$
\begin{aligned}
l_{1} & =\frac{e_{g-1} \bar{\beta}_{g}}{\beta_{0}}+\frac{1}{\mathrm{~m}\left(C_{1}\right)} \sum_{\Gamma \in \mathcal{B}}\left(C_{1}, \Gamma\right)_{0}=\frac{e_{g-1} \bar{\beta}_{g}}{\beta_{0}}+\frac{1}{\beta_{0}} \sum_{i=-1}^{g-1} a_{i} \bar{\beta}_{i+1} \\
& =\frac{e_{g-1} \bar{\beta}_{g}+\bar{\beta}_{g}+\sum_{i=-1}^{g-2} a_{i} \bar{\beta}_{i+1}}{\beta_{0}} .
\end{aligned}
$$

To compute $l_{\Gamma_{i}}$ for $i \geq 0$ observe that

$$
\max \left\{\eta\left(\Gamma_{i}\right), \max _{\Gamma \neq \Gamma_{i}} \frac{\left(\Gamma, \Gamma_{i}\right)_{0}}{\mathrm{~m}(\Gamma)}, \frac{\left(\Gamma_{i}, C_{1}\right)_{0}}{\mathrm{~m}\left(C_{1}\right)}\right\}=\frac{\bar{\beta}_{i+1}}{\beta_{0}} .
$$

Indeed, $\eta\left(\Gamma_{i}\right) \leq \bar{\beta}_{i+1} / \beta_{0}$ (by definition of $\left.\eta(\Gamma)\right)$, and $\left(\Gamma_{i}, C_{1}\right)_{0} / \mathrm{m}\left(C_{1}\right)=$ $\bar{\beta}_{i+1} / \beta_{0}$ and $\left(\Gamma, \Gamma_{i}\right)_{0} / \mathrm{m}(\Gamma) \leq \bar{\beta}_{i+1} / \beta_{0}$ by Property 4.2 . Therefore

$$
\begin{aligned}
l_{\Gamma_{i}} & =\frac{\bar{\beta}_{i+1}}{\beta_{0}}+\frac{e_{i} \bar{\beta}_{i+1}}{\beta_{0}}+\frac{1}{\mathrm{~m}\left(\Gamma_{i}\right)} \sum_{\Gamma \neq \Gamma_{i}}\left(\Gamma_{i}, \Gamma\right)_{0} \\
& \leq \frac{\bar{\beta}_{i+1}}{\beta_{0}}+\frac{e_{g-1} \bar{\beta}_{g}}{\beta_{0}}+\frac{1}{\mathrm{~m}\left(C_{1}\right)} \sum_{\Gamma \neq \Gamma_{i}}\left(C_{1}, \Gamma\right)_{0} \leq l_{1}
\end{aligned}
$$

by Property 4.2 and formula (27).

Now, suppose that $\mathcal{B}_{-1} \neq 0$ and let $\Gamma_{-1} \in \mathcal{B}_{-1}$. Since the branch $\left(\Gamma_{-1}, 0\right)$ is smooth, we have $\eta\left(\Gamma_{-1}\right)=-\infty$ and by $(24)$ we get

$$
\begin{aligned}
l_{\Gamma_{-1}}= & \max \left\{\max _{\Gamma \neq \Gamma_{-1}} \frac{\left(\Gamma_{-1}, \Gamma\right)_{0}}{\mathrm{~m}(\Gamma)}, \frac{\left(\Gamma_{-1}, C_{1}\right)_{0}}{\mathrm{~m}\left(C_{1}\right)}\right\} \\
& +\frac{\left(\Gamma_{-1}, C_{1}\right)_{0}+\sum_{\Gamma \neq \Gamma_{-1}}\left(\Gamma_{-1}, \Gamma\right)_{0}}{\mathrm{~m}\left(\Gamma_{-1}\right)} \\
= & 1+\sum_{\Gamma \neq \Gamma_{-1}} \mathrm{~m}(\Gamma)+\mathrm{m}\left(C_{1}\right)=\mathrm{m}\left(C_{1} \cup \bigcup_{i=-1}^{g-1} \mathcal{B}_{i}\right) \leq l_{1} .
\end{aligned}
$$

Proposition 4.1 follows from (25) and (27)-(29).

We complete Theorem 3.2 by the following result:

THEOREM 4.3. Let $\left(\beta_{0}, \ldots, \beta_{g}\right), g>0$, be a characteristic sequence and let $\delta=a_{-1} \bar{\beta}_{0}+a_{0} \bar{\beta}_{1}+\cdots+a_{g-2} \bar{\beta}_{g-1} \in\left\langle\bar{\beta}_{0}, \ldots, \bar{\beta}_{g-1}\right\rangle$ be such that $a_{i}=0$ or $a_{i}=1$ for $0 \leq i<g-2$. Then there exists a plane curve singularity $(C, 0)$ 
with a local equation $f=0$ for which there is a decomposition into branches $(C, 0)=\bigcup_{i=1}^{r}\left(C_{i}, 0\right), r>1$, such that:

(i) The branches $\left(C_{1}, 0\right)$ and $\left(C_{2}, 0\right)$ are of characteristic $\left(\beta_{0}, \ldots, \beta_{g}\right)$ and $\left(\beta_{0} / e_{g-1}, \ldots, \beta_{g-1} / e_{g-1}\right)$, respectively, and $\left(C_{1}, C_{2}\right)_{0}=\bar{\beta}_{g}$.

(ii) For every $i \neq 1,2$,

$$
\frac{\left(C_{1}, C_{i}\right)_{0}}{\mathrm{~m}\left(C_{1}\right) \mathrm{m}\left(C_{i}\right)}=\frac{\left(C_{2}, C_{i}\right)_{0}}{\mathrm{~m}\left(C_{2}\right) \mathrm{m}\left(C_{i}\right)}<\frac{\left(C_{1}, C_{2}\right)_{0}}{\mathrm{~m}\left(C_{1}\right) \mathrm{m}\left(C_{2}\right)} .
$$

(iii) $\mathcal{L}_{0}(f)+1=\left(e_{g-1} \bar{\beta}_{g}+\bar{\beta}_{g}+\delta\right) / \beta_{0}$ and $\delta=\sum_{i \neq 1,2}\left(C_{1}, C_{i}\right)_{0}$.

Proof. Let $\left(C_{1}, 0\right)$ be a branch with characteristic $\left(\beta_{0}, \ldots, \beta_{g}\right)$. By Remark 2.3 there exists a finite family of finite sets $\mathcal{B}_{i}$ of branches $(i=-1,0, \ldots, g-1)$ such that the assumptions of Proposition 4.1 are satisfied with $\sharp \mathcal{B}_{i}=a_{i}$. Let $\left(C_{2}, 0\right)$ be the unique branch of the family $\mathcal{B}_{g-1}$ and let $\left(C_{j}, 0\right), j \in\{3,4, \ldots, r\}$, be the branches of $\bigcup_{i=-1}^{g-2} \mathcal{B}_{i}$. Let $(C, 0)=\bigcup_{i=1}^{r}\left(C_{i}, 0\right)$. Using Proposition 4.1 we check that the three assertions of the theorem hold true.

EXAMPLE 4.4. Let $p>2$ be a prime number. Then $\lambda=(p+1)^{2}-1 / p^{2}$ is a nonregular Łojasiewicz number. Indeed, let $\left(\beta_{0}, \beta_{1}, \beta_{2}\right)=\left(p^{2}, p^{2}+p\right.$, $\left.p^{2}+2 p-1\right)$ and $\delta=0$. By Theorem 4.3 there is a plane singularity $f=0$ (with two branches) such that

$$
\mathcal{L}_{0}(f)+1=\frac{e_{1} \bar{\beta}_{2}+\bar{\beta}_{2}}{\beta_{0}} .
$$

Obviously $\mathcal{L}_{0}(f)=\lambda$ and $\mathcal{L}_{0}(f)$ is nonregular.

5. Arithmetical lemmas. Let $\left(\beta_{0}, \ldots, \beta_{g}\right)$ be a characteristic sequence. Recall that a rational number $\lambda$ is associated with $\left(\beta_{0}, \ldots, \beta_{g}\right)$ if there is an integer $\delta \in\left\langle\bar{\beta}_{0}, \ldots, \bar{\beta}_{g-1}\right\rangle$ such that

$$
\lambda+1=\frac{e_{g-1} \bar{\beta}_{g}+\bar{\beta}_{g}+\delta}{\beta_{0}} .
$$

Let $\lambda=N+b / a, 0 \leq b<a, \operatorname{GCD}(a, b)=1$, be a number associated with $\left(\beta_{0}, \ldots, \beta_{g}\right)$. We say that $\lambda$ is regular (resp. nonregular) if $a+b \leq N$ (resp. $a+b>N)$.

Lemma 5.1. Suppose that $\lambda=N+b / a, 0<b<a, \operatorname{GCD}(a, b)=1$, is a nonregular number associated with a characteristic sequence $\left(\beta_{0}, \ldots, \beta_{g}\right)$. Then

(i) $\beta_{1}<\lambda+1<2 \beta_{0}$,

(ii) $a=\beta_{0}$,

(iii) $g \geq 2$. 
Proof. It is easy to see that $\lambda+1<2 a$. If $\lambda$ is associated with $\left(\beta_{0}, \ldots, \beta_{g}\right)$ then obviously $a \leq \beta_{0}$ and we get $\lambda+1<2 \beta_{0}$. On the other hand,

$$
\lambda+1 \geq \frac{e_{g-1} \bar{\beta}_{g}+\bar{\beta}_{g}}{\beta_{0}}>\frac{e_{0} \bar{\beta}_{1}}{\beta_{0}}=\bar{\beta}_{1}=\beta_{1},
$$

which proves (i).

To check (ii) observe that if we had $a<\beta_{0}$ then the fraction $\lambda+1=$ $\left(e_{g-1} \bar{\beta}_{g}+\bar{\beta}_{g}+\delta\right) / \beta_{0}$ would simplify and we would get $a \leq \beta_{0} / d \leq \beta_{0} / 2$ (where $d>1$ is a divisor of $\beta_{0}$ ) and $\lambda+1<2 a \leq \beta_{0}$, which is impossible by (i).

Now we check (iii). To this end we have to show that any number $\lambda$ associated with the sequence $\left(\beta_{0}, \beta_{1}\right)$ is regular. We get $\lambda=\beta_{1}+a_{-1}+$ $\beta_{1}-\beta_{0} / \beta_{0}$ with an integer $a_{-1} \geq 0$. If $\beta_{1}-\beta_{0}>\beta_{0}$ the integral part of $\lambda$ is greater than $2 \beta_{0}$, while if $\beta_{1}-\beta_{0}<\beta_{0}$ then $\left(\beta_{1}-\beta_{0}\right)+\beta_{0}=\beta_{1} \leq[\lambda]$. In both cases $\lambda$ is a regular number.

LEMMA 5.2. Let $\left(\beta_{0}, \ldots, \beta_{g}\right)$ be a characteristic sequence such that there exists a nonregular number associated with it. Then

(i) $\left(e_{g-1}+1\right) \bar{\beta}_{g}<2 \beta_{0}^{2}$,

(ii) $\beta_{1}+\frac{\beta_{1}}{e_{1}}+\frac{\left(e_{1}+1\right)\left(\beta_{2}-\beta_{1}\right)}{\beta_{0}}<2 \beta_{0}$,

(iii) $\frac{\beta_{0}}{e_{1}}<\frac{\beta_{1}}{e_{1}}<\frac{2 \beta_{0}}{e_{1}+1}$.

Proof. The first condition follows from the inequality

$$
\lambda+1 \geq \frac{\left(e_{g-1}+1\right) \bar{\beta}_{g}}{\beta_{0}}
$$

and 5.1(i). To get (ii) we use (i) and the inequality $\left(e_{1}+1\right) \bar{\beta}_{2} \leq\left(e_{g-1}+1\right) \bar{\beta}_{g}$, which holds for $g \geq 2$. Finally, (iii) follows from (ii).

Proposition 5.3. If $\lambda=N+b / a, 0<b<a, \operatorname{GCD}(a, b)=1$, is a nonregular number associated with a characteristic sequence, then $a$ is a composite number strictly greater than 8 and $N \geq a+6$.

Proof. The number $\lambda=N+b / a$ is associated with a characteristic sequence $\left(\beta_{0}, \ldots, \beta_{g}\right)$ such that $\beta_{0}=a$ and $g \geq 2$. Therefore $a$ is not a prime number (if $\beta_{0}=a$ is a prime then $g=1$ ). To check that $a>8$ it suffices to prove that the numbers associated with characteristic sequences $\left(\beta_{0}, \ldots, \beta_{g}\right)$ such that $\beta_{0} \in\{4,6,8\}$ are regular. We may assume that $g \geq 2$. If $\beta_{0}=4$ then $e_{1}=2$ and condition (iii) of Lemma 5.2 is not satisfied because there is no integer in the interval $\left(\beta_{0} / e_{1}, 2 \beta_{0} /\left(e_{1}+1\right)\right)=\left(2,2 \frac{2}{3}\right)$. If $\beta_{0}=6$ then $e_{1}=2$ or $e_{1}=3$ and in both cases the interval $\left(\beta_{0} / e_{1}, 2 \beta_{0} /\left(e_{1}+1\right)\right)$ does 
not contain integers. Let $\beta_{0}=8$. Then $e_{1}=4$ or $e_{1}=2$. If $\beta_{0}=8$ and $e_{1}=4$ then condition (iii) of Lemma 5.2 gives $\beta_{1}=12$. Obviously $g \leq 3$. If $g=3$ then $\left(\beta_{0}, \ldots, \beta_{3}\right)=\left(8,12, \beta_{2}, \beta_{3}\right)$ where $\beta_{2}>12$ and $\beta_{2} \equiv 0(\bmod 2)$, that is, $\beta_{2} \geq 14$. It is easy to check that condition (ii) of Lemma 5.1 is not satisfied and consequently every number associated with $\left(8,12, \beta_{2}, \beta_{3}\right)$ is regular.

Suppose now that $g=2$. Then $\left(\beta_{0}, \beta_{1}, \beta_{2}\right)=\left(8,12, \beta_{2}\right)$ where $\beta_{2} \not \equiv 0$ $(\bmod 2)$ and $\beta_{2}>12$. Condition (ii) of Lemma 5.1 is satisfied only if $\beta_{2}=13$. We check easily that the numbers associated with the sequence $(8,12,13)$ are regular. Similarly a sequence $\left(\beta_{0}, \beta_{1}, \beta_{2}\right)$ with $\beta_{0}=8$ and $e_{1}=2$ satisfies the conditions of Lemma 5.2 if $\left(\beta_{0}, \beta_{1}, \beta_{2}\right)=(8,10,11)$. Every number associated with the sequence $(8,10,11)$ is regular. This proves the first part of the proposition.

Let $a>8$ be a composite number and let $\lambda=N+b / a$ be a nonregular Łojasiewicz exponent associated with the sequence $\left(\beta_{0}, \ldots, \beta_{g}\right)$. Then $\beta_{0}=a$ and $g \geq 2$ by Lemma 5.1. From the formula for $\lambda$ we get $\lambda \geq \beta_{1}-1+\beta_{1} / e_{1}$ and consequently $N \geq \beta_{1}-1+\beta_{1} / e_{1}$. If $\beta_{1} \geq a+5$ then the last inequality gives $N \geq a+6$, for $\beta_{1} / e_{1}$ is an integer greater than or equal to 2 . It suffices to consider the cases $\beta_{1} \in\{a+2, a+3, a+4\}$.

If $\beta_{1}=a+2$ then $e_{1}=2$ and

$$
N \geq(a+2)-1+\frac{a+2}{2} \geq a+6 \quad \text { for } a \equiv 0(\bmod 2) \text { and } a \neq 4,6 .
$$

If $\beta_{1}=a+3$ then $e_{1}=3$ and

$N \geq(a+3)-1+\frac{a+3}{3}=a+3+\frac{a}{3} \geq a+b \quad$ for $a \equiv 0(\bmod 3)$ and $a \geq 9$.

If $\beta_{1}=a+4$ then $e_{1}=2$ or $e_{1}=4$. If $\beta_{1}=a+4$ and $e_{1}=2$ then

$$
N \geq(a+3)+\frac{a+4}{2}=a+5+\frac{a}{2} \geq a+6 \quad \text { for } a \equiv 0(\bmod 2) .
$$

If $\beta_{1}=a+4$ and $e_{1}=4$ then

$$
N \geq(a+3)+\frac{a+4}{4}=a+4+\frac{a}{4} \geq a+6 \quad \text { for } a \equiv 0(\bmod 6) \text { and } a \neq 4 .
$$

Lemma 5.4. For every composite integer a $>8$ except 12, 14, 15, 20, there exists an integer $c>0$ such that

$$
p+\frac{a}{p}<(p+1) c<a \quad \text { and } \operatorname{GCD}(a, c)=1,
$$

where $p>1$ is the smallest prime divisor of a.

Proof. For every composite integer $a, 8<a \leq 30$, except 12, 14, 15, 20, we give a specific $c$. Namely, one can easily check that the following pairs $(a, c)$ fulfil $(30):(9,2),(10,3),(16,5),(18,5),(21,4),(22,5),(24,5),(25,2)$, $(26,7),(27,4),(28,9),(30,7)$. 
For every composite integer $a>30$ we apply induction with respect to the number of prime factors of $a$.

Let $a=p p_{1} \cdots p_{r}>30, r \geq 1$, be a factorization of $a$ into prime factors such that $p \leq p_{1} \leq \cdots \leq p_{r}$. The condition (30) means that we should find an integer

$$
c \in\left(\frac{p+p_{1} \cdots p_{r}}{p+1}, \frac{p p_{1} \cdots p_{r}}{p+1}\right)
$$

coprime to $p p_{1} \cdots p_{r}$. The length of this segment is equal to

$$
l=p_{1} \cdots p_{r} \frac{p-1}{p+1}-\frac{p}{p+1} .
$$

So, it suffices to find an integer $c$ coprime to $p p_{1} \cdots p_{r}$ in each open segment $(A, A+l)$ for every $A>0$. We do this by induction with respect to $r$.

$1^{\circ} . r=1$. Since $a=p p_{1}>30$ it follows that

(i) if $p=2$ then $p_{1} \geq 17$,

(ii) if $p=3$ then $p_{1} \geq 11$,

(iii) if $p \geq 5$ then $p_{1} \geq 7$.

In case (i) (resp. (ii), (iii)) the length $l$ of the segment is $>5$ (resp. $>4,>3$ ). In each segment of length $>5$ (resp. $>4,>3$ ) we can always find an integer coprime to 2 and $p_{1} \geq 17$ (resp. to 3 and $p_{1} \geq 11$, to $p \geq 5$ and $p_{1} \geq 7$ ).

$2^{\circ} . r \Rightarrow r+1$. Assume that for every $p p_{1} \cdots p_{r}>30, r \geq 1, p \leq p_{1} \leq$ $\ldots \leq p_{r}, p, p_{i}$ primes, and for every open segment $\left(A, A+p_{1} \cdots p_{r} \frac{p-1}{p+1}-\frac{p}{p+1}\right)$, $A>0$, there exists an integer $c$ belonging to this segment and coprime to $p p_{1} \cdots p_{r}$.

Take $a=p p_{1} \cdots p_{r+1}>30, p \leq p_{1} \leq \ldots \leq p_{r+1}, p, p_{i}$ primes and a segment $\left(A, A+p_{1} \cdots p_{r+1} \frac{p-1}{p+1}-\frac{p}{p+1}\right), A>0$. Put $p_{0}:=p$ and consider two cases:

(i) There exist $i, j \in\{0,1, \ldots, r+1\}, i<j$, such that $p_{i}=p_{j}$. Take the smallest such $i$. If $p_{0} p_{1} \cdots p_{j-1} p_{j+1} \cdots p_{r+1}>30$ then by induction hypothesis there exists an integer

$$
c \in\left(A, A+p_{1} \cdots p_{j-1} p_{j+1} \cdots p_{r+1} \frac{p_{0}-1}{p_{0}+1}-\frac{p_{0}}{p_{0}+1}\right)
$$

coprime to $p_{0} p_{1} \cdots p_{j-1} p_{j+1} \cdots p_{r+1}$. Since $p_{i}=p_{j}$, it follows that $c$ is coprime to $p_{0} p_{1} \cdots p_{r+1}$. Moreover,

$$
\begin{aligned}
\left(A, A+p_{1} \cdots p_{j-1} p_{j+1} \cdots p_{r+1}\right. & \left.\frac{p_{0}-1}{p_{0}+1}-\frac{p_{0}}{p_{0}+1}\right) \\
& \subset\left(A, A+p_{1} \cdots p_{r+1} \frac{p_{0}-1}{p_{0}+1}-\frac{p_{0}}{p_{0}+1}\right) .
\end{aligned}
$$

Hence $c$ satisfies the required conditions. 
If $p_{0} p_{1} \cdots p_{j-1} p_{j+1} \cdots p_{r+1} \leq 30$ then we easily check that $a$ is one of the following numbers: $32,36,40,44,45,48,50,52,54,56,60,63,75,81,90$, $98,147,150,242,338$. In each case we easily check that there exists $c$ with the required properties.

(ii) $p_{0}<p_{1}<\cdots<p_{r+1}$. Consider two subcases:

(a) $p_{0} p_{1} \cdots p_{r}>30$. Then by induction hypothesis applied to $p_{0} p_{1} \cdots p_{r}$, there exists an integer $\widetilde{c} \in\left(A, A+p_{1} \cdots p_{r} \frac{p_{0}-1}{p_{0}+1}-\frac{p_{0}}{p_{0}+1}\right)$ coprime to $p_{0} p_{1} \cdots p_{r}$. If $\widetilde{c}$ is also coprime to $p_{r+1}$ then $\widetilde{c}$ is the required number, since the above segment is contained in $\left(A, A+p_{1} \cdots p_{r+1} \frac{p_{0}-1}{p_{0}+1}-\frac{p_{0}}{p_{0}+1}\right)$. If $\widetilde{c}$ is not coprime to $p_{r+1}$ then $\widetilde{c}=k p_{r+1}$ for some $k \in \mathbb{N}$. Obviously $k$ is coprime to $p_{0} p_{1} \cdots p_{r}$. We put $c:=\widetilde{c}+p_{0} p_{1} \cdots p_{r}$ and we claim that $c$ satisfies the required conditions. Obviously $c$ is coprime to $p_{0} p_{1} \cdots p_{r+1}$. Moreover $c \in\left(A, A+p_{1} \cdots p_{r+1} \frac{p_{0}-1}{p_{0}+1}-\frac{p_{0}}{p_{0}+1}\right)$. In fact, since $c>\widetilde{c}$, it suffices to check that $c<A+p_{1} \cdots p_{r+1} \frac{p_{0}-1}{p_{0}+1}-\frac{p_{0}}{p_{0}+1}$. To prove this we first show that

$$
p_{r+1} \geq \frac{p_{0}+1}{p_{0}-1} p_{0}+1 .
$$

In fact, if $p_{0}=2$ then $p_{r+1} \geq 7$ (because $r \geq 1, p_{0}<\cdots<p_{r+1}$ and $p_{0} p_{1} \cdots p_{r+1}>30$ ) and inequality (31) follows. If $p_{0} \geq 3$ then $p_{r+1} \geq p_{0}+4$ (because $r \geq 1$ and $p_{0}<\cdots<p_{r+1}$ ) and (31) also follows. From (31) we get

$$
\begin{aligned}
A+p_{1} \cdots p_{r+1} \frac{p_{0}-1}{p_{0}+1}-\frac{p_{0}}{p_{0}+1} & \geq A+p_{1} \cdots p_{r} \frac{p_{0}-1}{p_{0}+1}-\frac{p_{0}}{p_{0}+1}+p_{0} p_{1} \cdots p_{r} \\
& >\widetilde{c}+p_{0} p_{1} \cdots p_{r}=c .
\end{aligned}
$$

(b) $p_{0} p_{1} \cdots p_{r} \leq 30$. Since $p_{0} p_{1} \cdots p_{r+1}>30$, we only have the following cases:

1. $a=2 \cdot 3 \cdot p_{2}, \quad p_{2} \geq 7$,

2. $a=2 \cdot 5 \cdot p_{2}, \quad p_{2} \geq 7$,

3. $a=2 \cdot 7 \cdot p_{2}, \quad p_{2} \geq 11$,

4. $a=2 \cdot 11 \cdot p_{2}, \quad p_{2} \geq 13$

5. $a=2 \cdot 13 \cdot p_{2}, \quad p_{2} \geq 17$,

6. $a=3 \cdot 5 \cdot p_{2}, \quad p_{2} \geq 7$,

7. $a=3 \cdot 7 \cdot p_{2}, \quad p_{2} \geq 11$,

8. $a=2 \cdot 3 \cdot 5 \cdot p_{3}, \quad p_{3} \geq 7$.

In case 1 the length $l$ of the segment satisfies the inequality

$$
l=p_{1} p_{2} \frac{p_{0}-1}{p_{0}+1}-\frac{p_{0}}{p_{0}+1} \geq 3 \cdot 7 \cdot \frac{1}{3}-\frac{2}{3}>6 .
$$


It is easily seen that in any segment of length $>6$ one can find an integer coprime to 2,3 and $p_{2} \geq 7$. We consider the remaining cases similarly.

This ends the proof of the lemma.

6. Proofs. The proofs of our results presented in the introduction are based on the theorems proved in Sections 3 and 4 and on the arithmetical lemmas from Section 5.

We omit the proof of the following simple lemma:

LEMMA 6.1. Every singular branch of a nondegenerate singularity in Kouchnirenko's sense has exactly one characteristic pair.

Now we can give

Proof of Main Theorem 1. Let $N, a, b$ be integers such that $0<b<a$, $\operatorname{GCD}(a, b)=1$ and $a+b \leq N$. If $a+b<N$ then the function $f(x, y)=$ $y^{N+2}+x y^{N+1}+x^{a+1} y^{N-a-b}+x^{N}$ is nondegenerate in Kouchnirenko's sense and $\mathcal{L}_{0}(f)=N+b / a$ (to calculate $\mathcal{L}_{0}(f)$ one can use [L, Theorem 1]). If $a+b=N$ then we take $f(x, y)=y^{a+1}+y x^{N}$.

Suppose that $f$ defines a nondegenerate singularity at $0 \in \mathbb{C}^{2}$. If the Łojasiewicz number $\mathcal{L}_{0}(f)$ has the denominator less than or equal to $\frac{1}{2} \mathrm{~m}(C)$ then $\mathcal{L}_{0}(f)$ is regular by Lemma 3.1. If $\mathcal{L}_{0}(f)$ has large denominator then by Corollary 3.3, $\mathcal{L}_{0}(f)$ is associated with the characteristic of a singular branch of $(C, 0)$. Thus $\mathcal{L}_{0}(f)$ is regular by Lemmas 6.1 and 5.1.

LEMMA 6.2. Every Lojasiewicz nonregular number $\lambda$ is associated with a characteristic, i.e. $\lambda+1=\left(e_{g-1} \bar{\beta}_{g}+\bar{\beta}_{g}+\delta\right) / \beta_{0}$ with $\delta \in\left\langle\bar{\beta}_{0}, \ldots, \bar{\beta}_{g-1}\right\rangle$.

Proof. Let $\lambda=\mathcal{L}_{0}(f)$ be a Łojasiewicz nonregular number. Then $\mathcal{L}_{0}(f)$ has a large denominator by Lemma 3.1 and is associated with the characteristic of a branch of $f=0$ by Corollary 3.3.

Proof of Main Theorem 2. Let $\lambda=N+b / a$ be a nonregular Łojasiewicz number. Then by Lemma 6.2 it is associated with a characteristic sequence. Now use Lemma 5.3.

The lemma below follows immediately from Theorem 4.3.

Lemma 6.3. Let $\left(\beta_{0}, \ldots, \beta_{g}\right), g>0$, be a characteristic sequence and let $\delta=a_{-1} \bar{\beta}_{0}+a_{0} \bar{\beta}_{1}+\cdots+a_{g-2} \bar{\beta}_{g-1}$ with $a_{i}=0$ or $a_{i}=1$ for $i \geq 0$. Then $\lambda$ defined by

is a Eojasiewicz number.

$$
\lambda+1=\frac{e_{g-1} \bar{\beta}_{g}+\bar{\beta}_{g}+\delta}{\beta_{0}}
$$

Proof of Main Theorem 3. Let $a>8$ be a composite integer. Consider three cases: 
1. $a=14,15,20$. By Lemma 6.3 one can easily check that $24 \frac{13}{14}, 24 \frac{13}{15}$, $33 \frac{19}{20}$ are nonregular Łojasiewicz numbers associated with the characteristic sequences $(14,16,25),(15,18,25)$ and $(20,22,35)$ for $\delta=0$, respectively.

2. $a \neq 14,15,20$ and $a \neq \equiv 0(\bmod 6)$. Let $c$ be an integer as in Lemma 5.4. Then by Lemma 6.3 for the characteristic sequence $(a, a+p, a+p+c)$ and $\delta=0$ we deduce that

$$
\lambda=a+p+\frac{a}{p}+\frac{(p+1) c}{a}
$$

is a Łojasiewicz number. It is a nonregular Łojasiewicz number with denomiator $a$.

3. $a \equiv 0(\bmod 6)$. By Lemma 6.3 for the characteristic sequence $\left(\beta_{0}, \beta_{1}, \beta_{2}\right)=(a, a+2, a+a / 3+1)$ and $\delta=a+2=\bar{\beta}_{1}$ we find that

$$
\lambda=\frac{3 a}{2}+3+\frac{a-1}{a}
$$

is a Łojasiewicz number. It is a nonregular Łojasiewicz number with denominator $a$.

This ends the proof of the theorem.

Finally, let us prove the property of Łojasiewicz numbers mentioned at the beginning of the introduction.

Proposition 6.4. Every Lojasiewicz number $\lambda \notin \mathbb{N}$ can be written in the form $\lambda=N+b / a, a, b, N \in \mathbb{N}, 0<b<a<N$.

Proof. If $a+b \leq N$ then the assertion is obvious. Suppose that $\lambda$ is a nonregular Łojasiewicz number. By Lemma 6.1, $\lambda$ is associated with a characteristic sequence $\left(\beta_{0}, \ldots, \beta_{g}\right)$. Suppose that $\operatorname{GCD}(a, b)=1$. Then by Lemma 5.1 we get $a=\beta_{0}$ and $N>\beta_{0}$ for $\lambda>\beta_{1}-1 \geq \beta_{0}$. Thus $a<N$.

7. Remarks and examples. 1. It is not easy to determine successive nonregular Łojasiewicz numbers. We will show that $\lambda_{0}=15 \frac{8}{9}$ is the smallest nonregular Łojasiewicz number. Putting $p=3$ in Example 4.4 we find that $15 \frac{8}{9}$ is a Łojasiewicz number. Let $\lambda=N+b / a, a, b, N \in \mathbb{N}, 0<b<a$, $\operatorname{GCD}(a, b)=1$, be a nonregular Łojasiewicz number. We have to check that $\lambda \geq 15 \frac{8}{9}$. If $a>9$ then $\lambda>16$ by Main Theorem 2. Let $a=9$. Since $\lambda$ is nonregular, it is associated (by Lemma 6.2) with a characteristic sequence $\left(\beta_{0}, \beta_{1}, \beta_{2}\right)$ with $\beta_{0}=9$. Using Lemma 5.2 we check that $\left(\beta_{0}, \beta_{1}, \beta_{2}\right)=(9,12,13)$ or $(9,12,14)$ or $(9,12,16)$ and that the numbers associated with these sequences are $15 \frac{4}{9}, 16 \frac{4}{9}, \ldots ; 15 \frac{8}{9}, 16 \frac{8}{9}, \ldots ; 16 \frac{7}{9}, \ldots$, respectively. Thus $\lambda \geq 15 \frac{8}{9}$ provided $\lambda$ is nonregular. 
2. To solve the problem stated in the introduction it suffices to describe the Łojasiewicz numbers associated with characteristic sequences. More specifically, it would suffice to give for every characteristic sequence $\left(\beta_{0}, \beta_{1}, \ldots, \beta_{g}\right)$ an effective description of all $\delta \in\left\langle\bar{\beta}_{0}, \ldots, \bar{\beta}_{g-1}\right\rangle$ for which $\left(e_{g-1} \bar{\beta}_{g}+\bar{\beta}_{g}+\delta\right) / \beta_{0}-1$ is a Łojasiewicz number.

3. B. Teissier proposed the following application of the main result. Let $\mathcal{O}=\mathcal{O}_{\mathbb{C}^{2}, 0}$ be the ring of holomorphic function germs at $0 \in \mathbb{C}^{2}$. An ideal $I \subset \mathcal{O}$ is called a jacobian ideal if there exists a holomorphic function germ $f$ with isolated critical point at 0 such that $I=(\partial f / \partial x, \partial f / \partial y)$ in $\mathcal{O}$. From Main Theorem 2 we easily obtain examples of nonjacobian ideals.

EXAmple 7.1. Using Main Theorem 2 we can find rational numbers $N+b / a, 0<b<a<N, a+b>N$, which are not Łojasiewicz numbers. Then the ideals $I=\left(x^{a+1}-y^{a}, x^{N-b} y^{b}\right) \subset \mathcal{O}$ are not jacobian ideals. Indeed, suppose to the contrary that there exists a holomorphic function germ $f$ such that $I=(\partial f / \partial x, \partial f / \partial y)$ in $\mathcal{O}$. One checks that it would imply $\mathcal{L}_{0}(f)=\mathcal{L}_{0}(I)=N+b / a$ (see [P1]), which contradicts the choice of $N+b / a$.

\section{References}

[Cha-Kra] J. Chądzyński and T. Krasiński, The Eojasiewicz exponent for analytic curves, in: Singularities Symposium- —ojasiewicz 70, Banach Center Publ. 44, Inst. Math., Polish Acad. Sci., Warszawa, 1998, 73-80.

[Ch-P] J. Chądzyński and A. Płoski, An inequality for intersection multiplicity of analytic curves, Bull. Polish Acad. Sci. Math. 36 (1988), 113-117.

[Ga-P] E. García Barroso and A. Płoski, On the Eojasiewicz numbers, C. R. Acad. Sci. Paris Sér. I 336 (2003), 585-588.

[Gw-P] J. Gwoździewicz and A. Płoski, On the approximate roots of polynomials, Ann. Polon. Math. 60 (1995), 199-210.

[K] A. G. Kouchnirenko, Polyèdres de Newton et nombres de Milnor, Invent. Math. 32 (1976), 1-31.

[L] A. Lenarcik, On the Eojasiewicz exponent of the gradient of a holomorphic function, in: Singularities Symposium — Łojasiewicz 70, Banach Center Publ. 44, Inst. Math., Polish Acad. Sci., Warszawa, 1998, 149-166.

[P1] A. Płoski, Multiplicity and the Eojasiewicz exponent, in: Singularities, Banach Center Publ. 20, PWN, Warszawa, 1988, 353-364.

[P2] - On the maximal polar quotient of an analytic plane curve, Kodai Math. J. 24 (2001), 120-133.

[S] H. J. S. Smith, On the higher singularities of plane curves, Proc. London Math. Soc. 6 (1875), 153-182.

[T] B. Teissier, Variétés polaires. I. Invariants polaires des singularités d'hypersurfaces, Invent. Math. 40 (1977), 267-292.

[Z1] O. Zariski, General theory of saturation and of saturated local rings II. Saturated local rings of dimension 1, Amer. J. Math. 93 (1971), 872-964. 
[Z2] O. Zariski, Le problème des modules pour les branches planes, Hermann, Paris, 1986.

Departamento de Matemática Fundamental

Faculty of Mathematics

Facultad de Matemáticas

University of Łódź

Universidad de La Laguna

Banacha 22

38271 La Laguna, Tenerife, España

90-238 Łódź, Poland

E-mail: ergarcia@ull.es

E-mail: krasinsk@krysia.uni.lodz.pl

Department of Mathematics

Technical University

Al. 1000 LPP 7

25-314 Kielce, Poland

E-mail: matap@tu.kielce.pl

Reçu par la Rédaction le 15.12.2004

Révisé le 2.4.2005 